\title{
A remark on the a-minimally thin sets associated with the Schrödinger operator
}

\section{Gaixian Xue*}

*Correspondence:

jingben84@163.com

School of Mathematics and Information Science, Henan

University of Economics and Law,

Zhengzhou, 450046, China

\section{Abstract}

The aim of this paper is to give a new criterion for a-minimally th sets at infinity with respect to the Schrödinger operator in a cone, which supple nen he results obtained by T. Zhao.

Keywords: minimally thin set; Schrödinger operato, reen a-py, ential

\section{Introduction and results}

Let $\mathbf{R}$ and $\mathbf{R}_{+}$be the set of all real numb and th, set of all positive real numbers, respectively. We denote by $\mathbf{R}^{n}(n \geq 2)$ the $n$ dim sional Euclidean space. A point in $\mathbf{R}^{n}$ is denoted by $P=\left(X, x_{n}\right), X=\left(x_{1}, x_{2}, \ldots, x_{n-1}\right)$. The Euclidean distance between two points $P$ and $Q$ in $\mathbf{R}^{n}$ is denoted by $|P-Q| \cdot \quad \mathbf{o}|P-O|$ with the origin $O$ of $\mathbf{R}^{n}$ is simply denoted by $|P|$. The boundary and the cure f a set $S$ in $\mathbf{R}^{n}$ are denoted by $\partial S$ and $\bar{S}$, respectively. Further, int $S, \operatorname{diam} S$, nd $\operatorname{dist}\left(S_{1}, S\right)$ stand for the interior of $S$, the diameter of $S$, and the distance between $C$ an , res pectively.

We introduc system o pherical coordinates $(r, \Theta), \Theta=\left(\theta_{1}, \theta_{2}, \ldots, \theta_{n-1}\right)$, in $\mathbf{R}^{n}$ which are related to carte $n$ coordinates $\left(x_{1}, x_{2}, \ldots, x_{n-1}, x_{n}\right)$ by $x_{n}=r \cos \theta_{1}$.

Let $D$ oe an arbitra $y$ domain in $\mathbf{R}^{n}$ and $\mathcal{A}_{a}$ denote the class of non-negative radial potentials $(P)$, i.e. $\mathrm{D} \leq a(P)=a(r), P=(r, \Theta) \in D$, such that $a \in L_{\text {loc }}^{b}(D)$ with some $b>n / 2$ if $n>4$ an $b=2$ if $n=2$ or $n=3$ (see [1, p.354] and [2]).

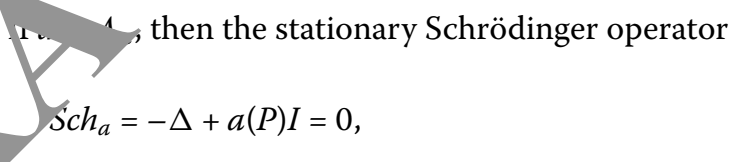

where $\Delta$ is the Laplace operator and $I$ is the identical operator, can be extended in the usual way from the space $C_{0}^{\infty}(D)$ to an essentially self-adjoint operator on $L^{2}(D)$ (see [1, Ch. 11]). We will denote it $S_{c h}$ as well. This last one has a Green $a$-function $G_{D}^{a}(P, Q)$. Here $G_{D}^{a}(P, Q)$ is positive on $D$ and its inner normal derivative $\partial G_{D}^{a}(P, Q) / \partial n_{Q} \geq 0$, where $\partial / \partial n_{Q}$ denotes the differentiation at $Q$ along the inward normal into $D$.

We call a function $u \neq \equiv-\infty$ that is upper semi-continuous in $D$ a subfunction with respect to the Schrödinger operator $S_{c h}$ if its values belong to the interval $[-\infty, \infty)$ and at each point $P \in D$ with $0<r<r(P)$ we have the generalized mean-value inequality (see [1, Ch. 11])

$$
u(P) \leq \int_{S(P, r)} u(Q) \frac{\partial G_{B(P, r)}^{a}(P, Q)}{\partial n_{Q}} d \sigma(Q)
$$

@2014 Xue; licensee Springer. This is an Open Access article distributed under the terms of the Creative Commons Attribution License (http://creativecommons.org/licenses/by/2.0), which permits unrestricted use, distribution, and reproduction in any medium, provided the original work is properly cited. 
satisfied, where $G_{B(P, r)}^{a}(P, Q)$ is the Green $a$-function of $S_{c h}$ in $B(P, r)$ and $d \sigma(Q)$ is a surface measure on the sphere $S(P, r)=\partial B(P, r)$. If $-u$ is a subfunction, then we call $u$ a superfunction (with respect to the Schrödinger operator $S c h_{a}$ ).

The unit sphere and the upper half unit sphere in $\mathbf{R}^{n}$ are denoted by $\mathbf{S}^{n-1}$ and $\mathbf{S}_{+}^{n-1}$, respectively. For simplicity, a point $(1, \Theta)$ on $\mathbf{S}^{n-1}$ and the set $\{\Theta ;(1, \Theta) \in \Omega\}$ for a set $\Omega$, $\Omega \subset \mathbf{S}^{n-1}$, are often identified with $\Theta$ and $\Omega$, respectively. For two sets $\Xi \subset \mathbf{R}_{+}$and $\Omega \subset \mathbf{S}^{n-1}$, the set $\left\{(r, \Theta) \in \mathbf{R}^{n} ; r \in \Xi,(1, \Theta) \in \Omega\right\}$ in $\mathbf{R}^{n}$ is simply denoted by $\Xi \times \Omega$. By $C_{n}(\Omega)$, we denote the set $\mathbf{R}_{+} \times \Omega$ in $\mathbf{R}^{n}$ with the domain $\Omega$ on $\mathbf{S}^{n-1}$. We call it a cone. W denote the set $I \times \Omega$ with an interval on $\mathbf{R}$ by $C_{n}(\Omega ; I)$.

From now on, we always assume $D=C_{n}(\Omega)$. For the sake of brevity, we sh 11 write $G_{\Omega}^{a}(P, Q)$ instead of $G_{C_{n}(\Omega)}^{a}(P, Q)$. We shall also write $g_{1} \approx g_{2}$ for two positi/re fi tions $g_{1}$ and $g_{2}$, if and only if there exists a positive constant $c$ such that $c^{-1} g_{1} \leq \widetilde{T}_{2} \leq$

Let $\Omega$ be a domain on $\mathbf{S}^{n-1}$ with smooth boundary. Consider the Di let prob $\mathrm{m}$

$$
\begin{aligned}
& \left(\Lambda_{n}+\lambda\right) \varphi=0 \quad \text { on } \Omega, \\
& \varphi=0 \quad \text { on } \partial \Omega,
\end{aligned}
$$

where $\Lambda_{n}$ is the spherical part of the Laplace operata $\Delta_{n}$

$$
\Delta_{n}=\frac{n-1}{r} \frac{\partial}{\partial r}+\frac{\partial^{2}}{\partial r^{2}}+\frac{\Lambda_{n}}{r^{2}}
$$

We denote the least positive eig nvalue this boundary value problem by $\lambda$ and the normalized positive eigenfunct on responding to $\lambda$ by $\varphi(\Theta)$. In order to ensure the existence of $\lambda$ and a smootb $\varphi(\theta)$, we $\mathrm{p} /$ a rather strong assumption on $\Omega$ : if $n \geq 3$, then $\Omega$ is a $C^{2, \alpha}$-domain $(0<<1)$ on $\mathbf{S}^{n-1}$ surrounded by a finite number of mutually disjoint closed hypersurfaces (e.

For any $(1, \Theta) \in$ ro have (see $[4, \mathrm{pp} .7-8]$ )

wh $P=(r, \Theta) \in C_{n}(\Omega)$ and $\delta(P)=\operatorname{dist}\left(P, \partial C_{n}(\Omega)\right)$.

Solu, ons of an ordinary differential equation (see $[5, \mathrm{p} .217])$

$$
-Q^{\prime \prime}(r)-\frac{n-1}{r} Q^{\prime}(r)+\left(\frac{\lambda}{r^{2}}+a(r)\right) Q(r)=0, \quad 0<r<\infty .
$$

It is well known (see, for example, [6]) that if the potential $a \in \mathcal{A}_{a}$, then equation (2) has a fundamental system of positive solutions $\{V, W\}$ such that $V$ and $W$ are increasing and decreasing, respectively.

We will also consider the class $\mathscr{B}_{a}$, consisting of the potentials $a \in \mathcal{A}_{a}$ such that there exists the finite $\operatorname{limit}_{\lim _{r \rightarrow \infty}} r^{2} a(r)=k \in[0, \infty)$, and, moreover, $r^{-1}\left|r^{2} a(r)-k\right| \in L(1, \infty)$. If $a \in \mathscr{B}_{a}$, then the (sub)superfunctions are continuous (see [7]). In the rest of paper, we assume that $a \in \mathscr{B}_{a}$ and we shall suppress this assumption for simplicity. 
Denote

$$
\iota_{k}^{ \pm}=\frac{2-n \pm \sqrt{(n-2)^{2}+4(k+\lambda)}}{2}
$$

then the solutions to equation (2) have the asymptotic (see [3])

$$
V(r) \approx r_{k}^{\iota_{k}^{+}}, \quad W(r) \approx r^{\iota_{k}^{-}}, \quad \text { as } r \rightarrow \infty
$$

It is well known that the Martin boundary of $C_{n}(\Omega)$ is the set $\partial C_{n}(\Omega) \cup\{\infty\}$, eacr of which is a minimal Martin boundary point. For $P \in C_{n}(\Omega)$ and $Q \in \partial C_{n}(\Omega) \cup \infty$, the Martin kernel can be defined by $M_{\Omega}^{a}(P, Q)$. If the reference point $P$ is chosen itat then we have

$$
M_{\Omega}^{a}(P, \infty)=V(r) \varphi(\Theta) \quad \text { and } \quad M_{\Omega}^{a}(P, O)=c W(r) \varphi(\Theta)
$$

for any $P=(r, \Theta) \in C_{n}(\Omega)$.

In [8, p.67], Zhao introduce the notations of a-thin ith respe $-t$ to the Schrödinger operator $S c h_{a}$ ) at a point, a-polar set (with respect to the $S c$ ir inger operator $S c h_{a}$ ) and a-minimal thin sets at infinity (with respect to the Schrödi ger operator $S c h_{a}$ ). A set $H$ in $\mathbf{R}^{n}$ is said to be a-thin at a point $Q$ if there a fin eighborhood $E$ of $Q$ which does not intersect $H \backslash\{Q\}$. Otherwise $H$ is said to he no thir at $Q$ on $C_{n}(\Omega)$. A set $H$ in $\mathbf{R}^{n}$ is called a polar set if there is a superfunction 1 on rome op n set $E$ such that $H \subset\{P \in E ; u(P)=\infty\}$. A subset $H$ of $C_{n}(\Omega)$ is said to be $-m$ nal th at $Q \in \partial C_{n}(\Omega) \cup\{\infty\}$ on $C_{n}(\Omega)$, if there exists a point $P \in C_{n}(\Omega)$ such a

$$
\hat{R}_{M_{\Omega}^{a}(\cdot, Q)}^{H}(P) \neq M_{\Omega}^{a}(, Q),
$$

where $\hat{R}_{M_{\Omega}^{a}(\cdot, Q)}^{H}$ is regularized reduced function of $M_{\Omega}^{a}(\cdot, Q)$ relative to $H$ (with respect to the Schrödinger $S$ pe, $_{\text {, tor }} \mathrm{Sch}_{a}$ ).

Let $H$ be c ounced subset of $C_{n}(\Omega)$. Then $\hat{R}_{M_{\Omega}^{a}(\cdot, \infty)}^{H}(P)$ is bounded on $C_{n}(\Omega)$ and hence the rrea ta-rarmonic minorant of $\hat{R}_{M_{\Omega}^{a}(\cdot, \infty)}^{H}$ is zero. When by $G_{\Omega}^{a} \mu(P)$ we denote the en a-po $y$ tial with a positive measure $\mu$ on $C_{n}(\Omega)$, we see from the Riesz decomposition orem that there exists a unique positive measure $\lambda_{H}^{a}$ on $C_{n}(\Omega)$ such that

$$
\hat{R}_{M_{\Omega}^{a}(\cdot, \infty)}^{H}(P)=G_{\Omega}^{a} \lambda_{H}^{a}(P)
$$

for any $P \in C_{n}(\Omega)$ and $\lambda_{H}^{a}$ is concentrated on $I_{H}$, where

$$
I_{H}=\left\{P \in C_{n}(\Omega) ; H \text { is not a-thin at } P\right\} .
$$

The Green a-energy $\gamma_{\Omega}^{a}(H)$ (with respect to the Schrödinger operator $S c h_{a}$ ) of $\lambda_{H}^{a}$ is defined by

$$
\gamma_{\Omega}^{a}(H)=\int_{C_{n}(\Omega)} G_{\Omega}^{a} \lambda_{H}^{a} d \lambda_{H}^{a} .
$$


Also, we can define a measure $\sigma_{\Omega}^{a}$ on $C_{n}(\Omega)$

$$
\sigma_{\Omega}^{a}(H)=\int_{H}\left(\frac{M_{\Omega}^{a}(P, \infty)}{\delta(P)}\right)^{2} d P
$$

In [8, Theorem 5.4.3], Long gave a criterion that characterizes a-minimally thin sets at infinity in a cone.

Theorem A $A$ subset $H$ of $C_{n}(\Omega)$ is a-minimally thin at infinity on $C_{n}(\Omega)$ if and only if

$$
\sum_{j=0}^{\infty} \gamma_{\Omega}^{a}\left(H_{j}\right) W\left(2^{j}\right) V^{-1}\left(2^{j}\right)<\infty
$$

where $H_{j}=H \cap C_{n}\left(\Omega ;\left[2^{j}, 2^{j+1}\right)\right)$ and $j=0,1,2, \ldots$

In recent work, Zhao (see [2, Theorems 1 and 2]) proved the following res. For similar results in the half space with respect to the Schrödinger oper ytor, re refer the reader to the papers by Ren and $\mathrm{Su}($ see $[9,10])$.

Theorem B The following statements are equivalent.

(I) A subset $H$ of $C_{n}(\Omega)$ is a-minimally thin at infinity o. $C_{n}(\Omega)$.

(II) There exists a positive superfunction $v\left(P, P_{n}(\Omega)\right.$ such that

$$
\inf _{P \in C_{n}(\Omega)} \frac{v(P)}{M_{\Omega}^{a}(P, \infty)}=0
$$

and

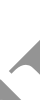

$$
H \subset\left\{P \in C_{n}(Q), y(P) \geq{ }^{a}(P, \infty)\right\} .
$$

(III) There exists a $p$ itive su ferfunction $v(P)$ on $C_{n}(\Omega)$ such that even if $v(P) \geq c M^{r a}(P, \infty)$ jor any $P \in H$, there exists $P_{0} \in C_{n}(\Omega)$ satisfying $v\left(P_{0}\right)<c M_{S}(1, \gamma)$

Theo, $\mathrm{n}$ If a suoset $H$ of $C_{n}(\Omega)$ is a-minimally thin at infinity on $C_{n}(\Omega)$, then we have

$$
\int_{Y} \frac{2}{(1+|P|)^{n}}<\infty
$$

Remark From equation (3), we immediately know that equation (6) is equivalent to

$$
\int_{H} V(1+|P|) W(1+|P|)(1+|P|)^{-2} d P<\infty .
$$

This paper aims to show that the sharpness of the characterization of an a-minimally thin set in Theorem C. In order to do this, we introduce the Whitney cubes in a cone.

A cube is the form

$$
\left[l_{1} 2^{-j},\left(l_{1}+1\right) 2^{-j}\right] \times \cdots \times\left[l_{n} 2^{-j},\left(l_{n}+1\right) 2^{-j}\right]
$$

where $j, l_{1}, \ldots, l_{n}$ are integers. The Whitney cubes of $C_{n}(\Omega)$ are a family of cubes having the following properties: 
(I) $\bigcup_{k} W_{k}=C_{n}(\Omega)$.

(II) int $W_{j} \cap$ int $W_{k}=\varnothing(j \neq k)$.

(III) $\operatorname{diam} W_{k} \leq \operatorname{dist}\left(W_{k}, \mathbf{R}^{n} \backslash C_{n}(\Omega)\right) \leq 4 \operatorname{diam} W_{k}$.

Theorem 1 If $H$ is a union of cubes from the Whitney cubes of $C_{n}(\Omega)$, then equation (7) is also sufficient for $H$ to be a-minimally thin at infinity with respect to $C_{n}(\Omega)$.

From the Remark and Theorem 1, we have the following.

Corollary 1 Let $v(P)$ be a positive superfunction on $C_{n}(\Omega)$ such that equation (5) notds. Then we have

$$
\int_{\left\{P \in C_{n}(\Omega) ;(P) \geq M_{\Omega}^{a}(P, \infty)\right\}} V(1+|P|) W(1+|P|)(1+|P|)^{-2} d P<\infty .
$$

Corollary 2 Let $H$ be a Borel measurable subset of $C_{n}(\Omega)$ satisf s.

$$
\int_{H} V(1+|P|) W(1+|P|)(1+|P|)^{-2} d P=+\infty \text {. }
$$

If $v(P)$ is a non-negative superfunction on $C_{n}(\Omega)$ and $c$ is a p ositive number such that $v(P) \geq$ $c M_{\Omega}^{a}(P, \infty)$ for all $P \in H$, then $v(P) \geq c M_{\Omega}^{a}(P \infty)_{\mathcal{\lambda}}$ all $P \in C_{n}(\Omega)$.

\section{Lemmas}

To prove our results, we need some la nas.

Lemma 1 Let $W_{k}$ be a cube rom independent of $k$ such $t^{1}$ at

$$
\gamma_{\Omega}^{a}\left(W_{k}\right) \leq c \sigma_{2}^{a}\left(W_{k}\right)
$$

Proof If pply cresult of Long (see [8, Theorem 6.1.3]) for compact set $\bar{W}_{k}$, we obtain a mea. $\mu$, $C, \Omega)$, supp $\mu \subset \bar{W}_{k}, \mu\left(\bar{W}_{k}\right)=1$ such that

$$
\begin{aligned}
& \left(\int_{C_{n}(\Omega)}|P-Q|^{2-n} d \mu(Q)=\left\{\operatorname{Cap}\left(\bar{W}_{k}\right)\right\}^{-1} \quad \text { if } n \geq 3,\right. \\
& C_{2}(\Omega) \log |P-Q| d \mu(Q)=\log \operatorname{Cap}\left(\bar{W}_{k}\right) \quad \text { if } n=2
\end{aligned}
$$

for any $P \in \bar{W}_{k}$. Also there exists a positive measure $\lambda \frac{a}{W_{k}}$ on $C_{n}(\Omega)$ such that

$$
\hat{R}_{M_{\Omega}^{a}(\cdot, \infty)}^{\bar{W}_{k}}(P)=G_{\Omega}^{a} \lambda \frac{a}{\bar{W}_{k}}(P)
$$

for any $P \in C_{n}(\Omega)$.

Let $P_{k}=\left(r_{k}, \Theta_{k}\right), \rho_{k}, t_{k}$ be the center of $W_{k}$, the diameter of $W_{j}$, the distance between $W_{k}$ and $\partial C_{n}(\Omega)$, respectively. Then we have $\rho_{k} \leq t_{k} \leq 4 \rho_{k}$ and $\rho_{k} \leq r_{k}$. Then from equation (1) we have

$$
r_{k} M_{\Omega}^{a}(P, \infty) \approx V\left(r_{k}\right) \rho_{k}
$$


for any $P \in \bar{W}_{k}$. We can also prove that

$$
G_{\Omega}^{a}(P, Q) \gtrsim \begin{cases}|P-Q|^{2-n} & \text { if } n \geq 3 \\ \log \frac{\rho_{k}}{|P-Q|} & \text { if } n=2\end{cases}
$$

for any $P \in \bar{W}_{k}$ and any $Q \in \bar{W}_{k}$. Hence we obtain

$$
r_{k} \lambda \frac{a}{W_{k}}\left(C_{n}(\Omega)\right) \lesssim \begin{cases}V\left(r_{k}\right) \rho_{k} \operatorname{Cap}\left(\bar{W}_{k}\right) & \text { if } n \geq 3, \\ V\left(r_{k}\right) \rho_{k}\left\{\log \frac{\rho_{k}}{\operatorname{Cap}\left(\bar{W}_{k}\right)}\right\}^{-1} & \text { if } n=2\end{cases}
$$

from equations (8), (9), (10), and (11). Since

$$
\left.\gamma_{\Omega}^{a}\left(W_{k}\right)=\int G_{\Omega}^{a} \lambda \frac{a}{\bar{W}_{k}} d \lambda \frac{a}{\bar{W}_{k}} \leq \int_{\bar{W}_{k}} M_{\Omega}^{a}(P, \infty) d \lambda \frac{a}{W_{k}}(P) \lesssim r_{k}^{l_{k}^{k}-1} \rho_{k} \lambda \frac{a}{W_{\mu}} C_{n}(\Omega)\right)
$$

from equations (3), (9), and (10), we have from (12)

$$
\gamma_{\Omega}^{a}\left(W_{k}\right) \lesssim \begin{cases}r_{k}^{2 l_{k}^{+}-2} \rho_{k}^{2} \operatorname{Cap}\left(\bar{W}_{k}\right) & \text { if } n \geq 3 \\ r_{k}^{2 l_{k}^{+}-2} \rho_{k}^{2}\left\{\log \frac{\rho_{k}}{\operatorname{Cap}\left(\bar{W}_{k}\right)}\right\}^{-1} & \text { if } n=2\end{cases}
$$

Since

$$
\begin{cases}\operatorname{Cap}\left(\bar{W}_{k}\right) \approx \rho_{k}^{n-2} & \text { if } n \geq 3 \\ \operatorname{Cap}\left(\bar{W}_{k}\right) \approx \rho_{k} & \text { if } n=2\end{cases}
$$

we obtain from equation $(13$,

$$
\gamma_{\Omega}^{a}\left(W_{k}\right) \lesssim r_{k}^{2 \iota^{+}-2} \rho
$$

On the other $h$

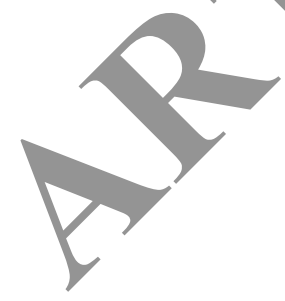

W. h, togetner with equation (14), gives the conclusion of Lemma 1.

\section{Proof of Theorem 1}

I $t\left\{W_{k}\right\}$ be a family of cubes from the Whitney cubes of $C_{n}(\Omega)$ such that $H=\bigcup_{k} W_{k}$. Let $\left\{W_{k, j}\right\}$ be a subfamily of $\left\{W_{k}\right\}$ such that $W_{k, j} \subset\left(H_{j-1} \cup H_{j} \cup H_{j+1}\right)$, where $j=1,2,3, \ldots$.

Since $\gamma_{\Omega}^{a}$ is a countably subadditive set function (see [8, p.49]), we have

$$
\gamma_{\Omega}^{a}\left(H_{j}\right) \lesssim \sum_{k} \gamma_{\Omega}^{a}\left(W_{k, j}\right)
$$

for $j=1,2, \ldots$ Hence for $j=1,2, \ldots$ we see from Lemma 1

$$
\sum_{k} \gamma_{\Omega}^{a}\left(W_{k, j}\right) \lesssim \sum_{k} \sigma_{\Omega}^{a}\left(W_{k, j}\right)
$$


which, together with equation (1), gives

$$
\begin{aligned}
\sum_{k} \sigma_{\Omega}^{a}\left(W_{k, j}\right) & \lesssim\left(\int_{H_{j-1}}+\int_{H_{j}}+\int_{H_{j+1}}\right) V^{2}(r) r^{-2} d P \\
& \lesssim\left(\int_{H_{j-1}}+\int_{H_{j}}+\int_{H_{j+1}}\right) r^{2\left(\iota_{k}^{+}-1\right)} d P \\
& \lesssim r^{2(j-1)\left(\iota_{k}^{+}-1\right)}\left|H_{j-1}\right|+r^{2 j\left(l_{k}^{+}-1\right)}\left|H_{j}\right|+r^{2(j+1)\left(\iota_{k}^{+}-1\right)}\left|H_{j+1}\right|
\end{aligned}
$$

for $j=1,2, \ldots$ Thus equations (15), (16), and (17) give

$$
\gamma_{\Omega}^{a}\left(H_{j}\right) \lesssim r^{2(j-1)\left(\iota_{k}^{+}-1\right)}\left|H_{j-1}\right|+r^{2 j\left(\iota_{k}^{+}-1\right)}\left|H_{j}\right|+r^{2(j+1)\left(\iota_{k}^{+}-1\right)}\left|H_{j+1}\right|
$$

for $j=1,2, \ldots$. Finally we obtain from equation (1)

$$
\begin{aligned}
\sum_{j=0}^{\infty} \gamma_{\Omega}^{a}\left(H_{j}\right) W\left(2^{j}\right) V^{-1}\left(2^{j}\right) & \lesssim \gamma_{\Omega}^{a}\left(H_{0}\right)+\sum_{j=0}^{\infty} 2^{j\left(2 l_{k}^{+}-2\right)} 2^{-j\left(l_{k}^{+}+l_{k}^{-}\right)} \\
& \lesssim \gamma_{\Omega}^{a}\left(H_{0}\right)+\left.\sum_{j=0}^{\infty} 2^{-2 j} W\left(2^{j}\right)\right|^{1} \mid \\
& \lesssim \gamma_{\Omega}^{a}\left(H_{0}\right)+\int_{H} \mathrm{~V}(1+\mid) W(1+|P|)(1+|P|)^{-2} d P \\
& <\infty,
\end{aligned}
$$

which shows with Theorem A at $H$ is a Mimally thin at infinity with respect to $C_{n}(\Omega)$.

The author declares that they ve no competing interests.

Acknowledgements

This work was support the National Natural Science Foundation of China under Grants Nos. 11301140 and U1304102.

Received 23 Fe uary 20 \% Accepted: 29 April 2014 Published: 23 May 2014

\section{Ref-ronces}

Levin, B, K MS, A: Asymptotic behavior of subfunctions of time-independent Schrödinger operator. In: Some vics on Value Distribution and Differentiability in Complex and P-Adic Analysis, Chap. 11, pp. 323-397. Science Beijing (2008)

2. Zhà T: Minimally thin sets at infinity with respect to the Schrödinger operator. J. Inequal. Appl. 2014, Article ID 67 (2014)

Gilbarg, D, Trudinger, NS: Elliptic Partial Differential Equations of Second Order. Springer, Berlin (1977)

Courant, R, Hilbert, D: Methods of Mathematical Physics, vol. 1. Interscience, New York (2008)

5. Miranda, C: Partial Differential Equations of Elliptic Type. Springer, London (1970)

6. Verzhbinskii, GM, Maz'ya, VG: Asymptotic behavior of solutions of elliptic equations of the second order close to a boundary. I. Sib. Mat. Zh. 12, 874-899 (1971)

7. Simon, B: Schrödinger semigroups. Bull. Am. Math. Soc. 7, 447-526 (1982)

8. Long, PH: The Characterizations of Exceptional Sets and Growth Properties in Classical or Nonlinear Potential Theory. Dissertation of Beijing Normal University, Beijing (2012)

9. Ren, YD: Solving integral representations problems for the stationary Schrödinger equation. Abstr. Appl. Anal. 2013, Article ID 715252 (2013)

10. Su, BY: Dirichlet problem for the Schrödinger operator in a half space. Abstr. Appl. Anal. 2012, Article ID 578197 (2012)

10.1186/1687-2770-2014-133

Cite this article as: Xue: A remark on the a-minimally thin sets associated with the Schrödinger operator. Boundary Value Problems 2014, 2014:133 